GUEST EDITORIAL

\title{
Bleeding disorders (part 1)
}

Haemostasis is a physiological process that stops blood loss at the site of injury, while maintaining normal blood flow in the rest of the circulation. This is accomplished in three physiological steps that occur in rapid sequence: (i) vasoconstriction; (ii) formation of a platelet plug (primary haemostasis); and (iii) stabilisation of clot through cross-linking of insoluble fibrin (secondary haemostasis). The fibrin mesh that is incorporated into and around the platelet plug serves to strengthen and stabilise the blood clot. Apart from limiting blood loss, the clot allows for vessel and tissue repair. Anticoagulant mechanisms regulate the coagulation system to ensure formation of a clot that is proportional to the injury. A delicate balance between procoagulant and anticoagulant systems is critical for proper haemostasis and for avoiding pathological bleeding or thrombosis. The clot is finally dissolved by the fibrinolytic system, which also performs the function of preventing blood clots in healthy blood vessels.

For the purpose of discussion in this two-part CME series, bleeding disorders are divided into two broad categories: (i) inherited (part 1, current issue); ${ }^{[1]}$ and (ii) acquired (part 2, forthcoming issue).

Inherited bleeding disorders (IBDs) should be suspected when there is a family history of a bleeding disorder or abnormal bleeding during early childhood, such as the neonatal period or infancy. However, IBD patients may also present for the first time during adulthood.

Investigation of abnormal bleeding requires a comprehensive history, thorough physical examination and systematic laboratory work-up. For the laboratory work-up, an algorithmic approach is included in the article in this edition of CME (Fig. 2). ${ }^{[1]}$

To appreciate the rationale for using the various clotting tests towards achieving a diagnosis, an understanding of the coagulation cascade is necessary. A simplified schematic representation - Fig. 1 in the article in this issue ${ }^{[1]}-$ is intended to orientate the reader with regard to the pathophysiology of the respective bleeding disorders.

The article discusses a wide range of IBDs and provides a diagnostic approach and available therapeutic options for the general practitioner. Patients diagnosed with a bleeding diathesis should ideally be referred to a tertiary healthcare facility such as a haemophilia centre for management and follow-up.

von Willebrand disease and haemophilia, being the most common IBDs, are discussed in greater detail. However, a comprehensive review of the various bleeding disorders with elaborate detail, such as pathophysiological mechanisms and genetic defects, is beyond the scope of this CME.

There has been significant progress with regard to the treatment of haemophilia, where the current standard of care is to prevent bleeding by prophylactic infusions of the deficient clotting factor as opposed to on-demand infusions following a bleed. This preventive strategy that is sure to reduce the hospitalisation and complication rates, comes at a cost, which is a limiting factor. Newer technologies with development of modified factor VIII products with an extended half-life and less immunogenic potential are emerging. With less frequent factor infusions, the prospect of prophylactic therapy is likely to become more affordable in economically challenged environments such as South Africa. This is an exciting yet realistic prospect that would allow haemophiliacs to lead almost normal lives. For any disease a cure is the ultimate aim, and in this context gene therapy has recently emerged as a potential therapeutic option for haemophilia B.

The authors are indeed grateful for the opportunity to discuss the subject of bleeding disorders, which is an important and dynamic sphere of coagulation.

\section{N Alli}

Department of Molecular Medicine and Haematology, School of Pathology, Faculty of Health Sciences, University of the Witwatersrand, Johannesburg, and National Health Laboratory Service, Johannesburg, South Africa nazeer.alli@nhls.ac.za

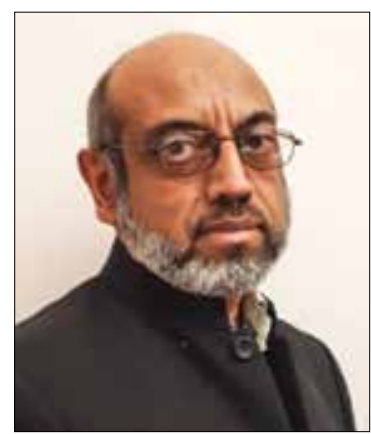

1. Alli N, Vaughan J, Louw S, Schapkaitz E, Mahlangu J. Inherited bleeding disorders. S Afr Med J 2018;108(1):9-15. DOI:10.7196/SAMI.2018.v108i1.13020.

S Afr Med J 2018;108(1):8. DOI:10.7196/SAMJ.2018.v108i1.13019 